\title{
ANALYTIC APPROXIMATION TO LOCALISED $A B$ INITIO PSEUDOPOTENTIAL AND SOME APPLICATIONS
}

\author{
A. SARKar* AND D. SEN ${ }^{\dagger}$ \\ Condensed Matter Physics Research Centre, Department of Physics \\ Jadavpur University \\ Jadavpur, Calcutta-700032, India \\ (Received September 16, 1996; revised version March 18, 1997)

\begin{abstract}
The localised version of the density functional based norm-conserving pseudopotential has already been applied to various metallic solids and aggregate state properties with some success. Applications to the study of liquid phonons and to the surface dynamics are specially discussed in the present paper. An analytic fit to the pseudopotential is obtained and further application to molecular dynamics is indicated.
\end{abstract}

PACS numbers: $63.10 .+a, 71.10 .-\mathrm{w}$

\section{Introduction}

Over the past decade, density functional based first principle calculations of both molecular dynamics [1,2] of clusters and extended systems and lattice mechanical properties of crystalline solids [3-6] on the basis of norm-conserving ab initio pseudopotentials (AP) [7] have claimed remarkable success. For the lattice mechanical properties the usual method employs a nonperturbative approach in the framework of local density functional technique. A self-consistent band structure calculation is performed with the pseudopotential derived following Hamann et al. scheme [7]. A more recent first principle calculational scheme using a generalized orthonormality condition for the generation of "ultrasoft" pseudopotential [8] has been successfully applied to molecular dynamics simulation [2]. Both these schemes give nonlocal pseudopotentials and require very accurate and time consuming numerical computations.

The nonlocality of the pseudopotential appears with the passage from the true ion-electron potential in a solid to the weak effective potential for the valence electrons. Actually, nonlocality is present due to the exchange of the conduction-

*B.K. Girls' College, Howrah 711 101, West Bengal, India.

†Barasat Govt. College, Barasat, West Bengal, India. 
and inner electrons even in the self-consistent "all electron" crystal potential. Ilowever, this potential is always conveniently approximated as local. In model (pseudo-) potential calculations of metallic properties both local and nonlocal versions are freely used. A close scrutiny of the results of various calculations shows that nothing specific is lost by the non-inclusion of explicit nonlocality [9, 10-15]. Following Heine and Weaire we note that nonlocality may be important for physical effects which depend on the oscillating part of the energy-wave number characteristic function (e.w.f.) $F(q)$ near $2 k_{\mathrm{F}}$, "though relatively litlle is known yet about how important. It is paradoxical, and not yet understood, that nonlocality reduces the magnitude of the up-and-down variation of e.w.f. (or the related Cochran function) by such large a factor, and yet quite sensible results have been obtained for phonon dispersion of $\mathrm{Al}$ using the on-Fermi sphere approximation". [16]. In other words, there exists no universal signature of nonlocality in specific solid state properties of metals like those of many body interactions, for example, evident in the violation of Cauchy relation or from the appearance of some special features in phonon dispersion $[11,15]$. On the other hand, there are indications [16] that the magnitude of nonlocal contributions might be exaggerated in some model potential calculations.

In the case of first principle pseudopotentials, however, the nonlocality assumes microscopic theoretical origin. But even then, it is not at all clear how important this nonlocality is in the calculation of the specific solid state properties. Firstly, the pseudopotential is nonunique, and secondly, the separation of local and nonlocal contributions is rather arbitrary. For example, in the case of AP the arbitrariness in the nonlocal contributions stems from different $r_{\mathrm{c}}$ values used for pseudo (atomic) wave functions of different $l$-values.

On the other hand, Starkloff and Joannopoulos [13] have shown that a local first principle pseudopotential may be generated even for $d$-band metals if we "include outermost $s$ and $p$ core electrons in addition to the customary $d$ and $s$ valence electrons" to provide a description of the complete outer (valence) shells. Later on, Manninen et al. [10] have also obtained a local first principle pseudopotential to calculate several properties of aluminium and subsequently used to construct the interionic potential and electron-phonon interaction.

This explicit nonlocal character of the pseudopotential is not essential, for a host of simple metallic properties, appears amply evident in a relatively recent formulation by Sen [9]. The author presented a scheme for determining the pseudopotential form factors using a local (beyond on Fermi sphere) approximation from the nonlocal AP of Bachlet et al. [7] and finally made a perturbative study for some simple metals using linear dielectric screening. The calculation saves a lot of computation compared to the nonperturbative nonlocal calculation and yet produces reasonable agreement with experimental values which suggests fair degree of reliability of the localised form factors. The approach, therefore, provides enough simplicity and widens the scope of applicability of the pseudopotential methods on one hand and justifies the use of effective local (pseudo-) potential for a number of metallic systems on the other. As for example, electronic transport properties calculated on the basis of this scheme provide a good account of the experimental data. 
In the present work we further test the reliability of this simplified scheme and compare the resulting pseudopotential with the ab initio and well tested model potentials in some specific problems. In the next section we discuss the calculational results of some properties which depend mainly on the pseudopotential form factor. Calculations are performed for: (i) band gaps, (ii) resistivity of vacancy and (iii) the Madelung constant for isotropic systems (liquid metals). Sarkar et al. [17] have subsequently constructed a real space local pseudopotential (numerical) from the Fourier transform of the numerical form factor [9]. The results of application of this potential in deriving effective ion-ion interaction and in the calculation of the frequency of collective excitation in liquid $R b$ are discussed. Finally, what we call a rationalized pseudopotential is obtained by fitting the numerical pseudopotential to an analytical form. The analytic form and its application in surface dynamics of metals is presented. The usefulness of the analytic form in molecular dynamics calculations is also indicated.

\section{Pseudopotential calculations of properties of metals}

The local approximation [9] makes an angular average of the nonlocal ab initio pseudopotential [7] form factors considering scattering in all possible directions (on and off Fermi sphere). The average form factor $V_{q}$ so obtained appears to retain the essential nonlocal effect in reducing the up-and-down variation near $2 k_{\mathrm{F}}$ of the function $F(q)$ (Fig. 1) as observed by Heine and Weaire [16]. We also note that the first-order perturbation energy which is simply the valence $(Z)$ times the non-Coulomb part of the form factor at $q=0$, agrees within $3 \%$ with the value of the exact nonlocal calculation. For example, the calculated value in the

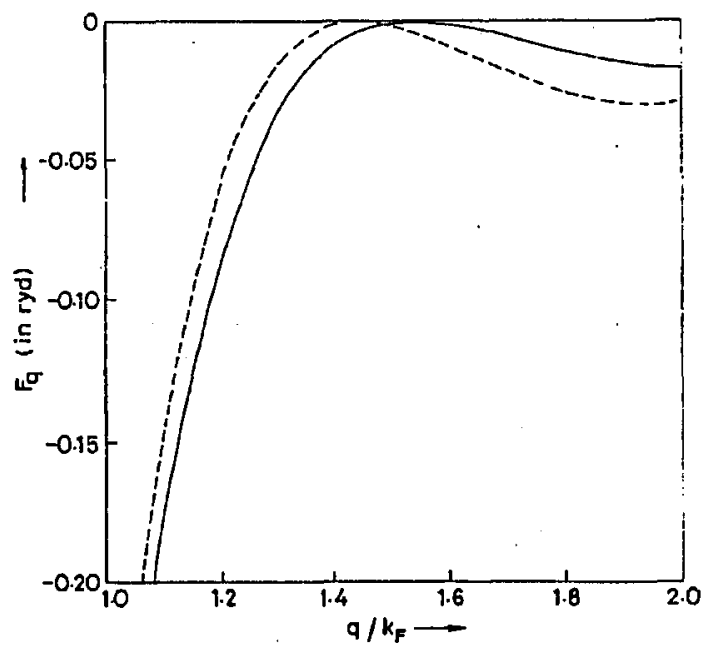

Fig. 1. $F(q)$ vs. $g$ for Al. The solid line represents the result with localised pseudopotential and the dashed curve represents the same with on-Frmi sphere approximation (OFSA) version. 
local approximation for $\mathrm{Al}$ is $1.42 \mathrm{Ry}$ compared to $1.47 \mathrm{Ry}$ of the exact nonlocal calculation [6].

Most of electronic properties are determined by the amplitude for the scattering of an electron by a single ion, i.e., the pseudopotential form factors. These include shape of the Fermi surface, band gaps, optical properties and transport properties of disordered materials such as liquid or amorphous metals and alloys.

\section{TABLE I}

The bare-ion pseudopotential form factors are compared with the $V(q)$ 's obtained directly from different experimental data.

\begin{tabular}{l|c|l|l|l}
\hline \hline Element & $G$ (or $q)^{a}$ & $G / 2 k_{\mathrm{F}}$ & \multicolumn{2}{|c}{$V(G)[\mathrm{Ry}]$} \\
\cline { 4 - 5 } & & & Calc. & Expt. data $^{b}$ \\
\hline $\mathrm{Na}$ & $(110)$ & 1.14 & 0.044 & $0.036(\mathrm{Opt})$ \\
& $(200)$ & 1.60 & 0.052 & $\mp 0.02(\mathrm{FS})$ \\
$\mathrm{Mg}$ & $q_{0}$ & 0.88 & 0.0 & $0.866(\mathrm{Opt})$ \\
& $(10 \overline{1} 0)$ & 0.829 & 0.008 & $0.014(\mathrm{FS})$ \\
& & & & $0.0118(\mathrm{Opt})$ \\
& $(0002)$ & 0.879 & 0.024 & $0.026(\mathrm{FS})$ \\
& & & & $0.0228(\mathrm{Opt})$ \\
& $(10 \overline{1} 1)$ & 0.937 & 0.040 & $0.036(\mathrm{FS})$ \\
& & & & $0.0309(\mathrm{Opt})$ \\
& $(10 \overline{1} 2)$ & 1.208 & 0.079 & $0.058(\mathrm{FS})$ \\
& & & & $0.0309(\mathrm{Opt})$ \\
& $q_{0}$ & 0.81 & 0.0 & $0.83(\mathrm{Opt})$ \\
$\mathrm{Al}$ & & & $0.78\left(\rho_{\mathrm{L}}\right)$ \\
& $(111)$ & 0.78 & 0.020 & $0.0179(\mathrm{FS})$ \\
& $(200)$ & 0.88 & 0.051 & $0.056(\mathrm{FS})$ \\
& $q_{0}$ & 0.765 & 0.0 & $0.76(\mathrm{Opt})$ \\
\hline
\end{tabular}

${ }^{a} q_{0}$ is the value of $q$ where $V(q)$ first passes through zero.

$b$ Type of experimental data fitted to as follows; FS $=$ Fermi surface, Opt $=$ optical spectrum; $\rho_{\mathrm{L}}=$ resistivity of liquid metal. All these experimental data are taken from Cohen and Heine [18].

In Table I a direct comparison of localised pseudopotential form factor $V_{q}$ with the empirical values [18] determined from various electronic properties is made for some simple metals. Calculation of electrical resistivity of liquid simple metals has already been shown $[9,19]$ to produce reasonably good agreement with the experiment. Here we present (Table II) the results of resistivity due to singly isolated vacancies in some simple metals for which the pseudopotential theory is 
TABLE II

Monovacancy resistivity of some simple metals.

\begin{tabular}{l|c|c|c|c}
\hline \hline Element & \multirow{2}{*}{$\begin{array}{r}r_{\mathrm{s}} \\
\text { [a.u.] }\end{array}$} & \multicolumn{3}{|c}{$\rho_{\mathrm{v}}[\mu \Omega \mathrm{cm} / \mathrm{at \%}]$} \\
\cline { 3 - 5 } & & $\begin{array}{c}\text { Present } \\
\text { calculation }\end{array}$ & \multicolumn{2}{|c}{ From Ref. } \\
\cline { 3 - 5 } & & {$[30]$} & {$[31]$} \\
\hline $\mathrm{Na}$ & 3.91 & 1.13 & 1.32 & 1.138 \\
$\mathrm{~K}$ & 4.85 & 1.99 & 1.66 & 1.531 \\
$\mathrm{Rb}$ & 5.19 & 3.02 & 1.84 & - \\
$\mathrm{Mg}$ & 2.63 & 0.73 & - & 0.767 \\
$\mathrm{Al}$ & 2.05 & 0.74 & 0.67 & 0.673
\end{tabular}

quite straightforward [20]. As no experimental data are yet available the calculated results are compared with some recent calculations.

Following Ashcroft [21] the Madelung energy of metallic system may be written as

$$
U_{\mathrm{M}}=\left(Z^{2} N / \Omega\right) \sum_{k=0}\left(S_{k}-1\right) V_{k}^{\mathrm{C}}+Z N \rho\left(V_{0}^{\mathrm{C}}-V_{0}^{\mathrm{P}}\right) \ldots
$$

with $\rho=(Z N / \Omega), S_{k}$ is the static structure factor and $V_{k}^{\mathrm{I}}$ 's are the corresponding Fourier transforms of the Coulomb and the pseudopotential where

$$
V_{k}^{\mathrm{I}}=4 \pi \int V^{\mathrm{I}}(r)(\sin k r / k r) r^{2} \mathrm{~d} r, \quad V_{0}^{\mathrm{I}}=\lim _{k \rightarrow 0} V_{k}^{\mathrm{I}} \ldots
$$

TABLE III

Madelung constant for liquid $\mathrm{Na}$ from pseudopotential calculations.

\begin{tabular}{c|c|c}
\hline $\begin{array}{c}\text { Standard value } \\
\text { of } \alpha=U_{\mathrm{M}} r_{\mathrm{s}} / e^{2}\end{array}$ & $\begin{array}{c}\alpha \text { from localised } \\
\text { pseudopotential }\end{array}$ & $\begin{array}{c}\text { From Heine-Abarenkov } \\
\text { model potential }^{a}\end{array}$ \\
\hline 1.80 & $\begin{array}{c}1.57 \\
\left(S_{\mathrm{K}} \text { from PY model }\right)\end{array}$ & $\begin{array}{c}1.02 \\
\left(R_{\mathrm{c}}=2.29 \text { a.u. }\right)\end{array}$ \\
\hline
\end{tabular}

${ }^{a}$ Ref. [11] (1983).

For the sake of simplicity we carried out the calculation for liquid $\mathrm{Na}$ for which the Madelung constant should agree with the standard value for any isotropic system. The results of our calculations are shown in Table III. For the localised pseudopotential the result is far better than the usual model potentials. For Ashcroft potential the result is very poor even with a large potential parameter $R_{\mathrm{c}}=$ 2.29 a.u., as $\alpha\left(=U_{\mathrm{M}} r_{\mathrm{s}} / e^{2}\right)$ turns out to be 0.6 , whereas the experimental transport properties indicate [22] that maximum permissible $R_{\mathrm{c}}$ value is 1.67 a.u. for $\mathrm{Na}$.

There are two other types of physical properties which can be calculated by the pseudopotential method - the so-called atomic properties. The first type demands the value of total crystal energy or some of its components. Apart from the usual crystal-structure energy calculations and the related crystal lattice stability studies this includes the static imperfections in crystals. 
The second type involves the properties associated with the way a solid responds to external influences. These properties are, therefore, determined by coordinate derivatives of the total energy rather than by magnitude. The phonon spectra, elastic constants and their pressure derivatives, Grüneisen's constants, equation of state, etc. are examples of this type. These properties are rather well studied with the localised $a b$ initio pseudopotentials.

\section{Some further applications}

The localised pseudopotential is also applied to investigate the temperature dependence of the dynamical structure, in particular the dispersion relation of the collective excitation (phonon-like) in the expanded liquid rubidium [23]. The effective ionic pair potential is derived by the second-order pseudopotential theory at different temperatures. Both static and dynamic structure factors are obtained from the effective pair potential and the dispersion relations of the collective excitation are in fair agreement with experiment for $350 \mathrm{~K}$ and $900 \mathrm{~K}$. At still higher temperatures the agreement is less encouraging but calculations including contribution from third order to the pair potential and with better dielectric screening show improvement and the work is in progress. In Fig. 2 the calculated results for static structure factor is compared with the results of modified hypernetted-chain (MHNC) approximation calculation [24].

The computed effective pair potentials show marked improvement over model pseudopotential calculations. The potential is relatively soft-core and both the position and the value of the potential minimum are closer to the empirical values [25] than existing model calculations. The minimum is roughly twice the value of the existing model calculations. A detailed study on effective two-body interaction with the localised $a b$ initio pseudopotential will be discussed elsewhere.

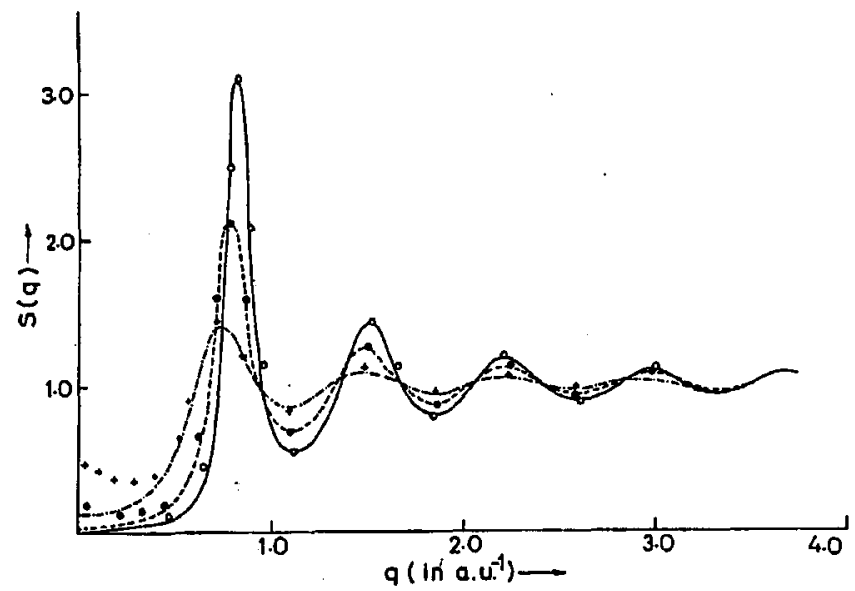

Fig. 2. Static structure factor $S_{q}$ of Rb. The solid line represents $T=350 \mathrm{~K}$, dashed curve $T=900 \mathrm{~K}$ and the dotted curve $T=1700 \mathrm{~K}$. The results of MINC calculation are indicated by discrete points. 
The computational scheme may further be simplified with an analytic fit to the numerical real space pseudopotential [26] (Fig. 3) obtained from the numerical form factors [9]. The core charge distribution according to various calculations is found to be of Gaussian type. In the limit $r \rightarrow 0$ the effective electron-ion potential is finite and as $r \rightarrow \infty$ it is pure Coulomb. Therefore the pseudopotential may be written as a direct Coulomb one and a part which is essentially a Gaussian function modulated by a polynomial in $r$

$$
V(r)=-Z e^{2}\left\{\left[1-\exp \left(-\alpha r^{2}\right)\right] / r+\left(A+B r+C r^{2}\right) \exp \left(-\alpha r^{2}\right)\right\} \ldots
$$

The four parameters $\alpha, A, B$ and $C$ are conveniently determined (shown in Table IV) from the numerical potential with following inputs:

(i) the value of the potential at $r=0$,

(ii) the first zero of the potential,

(iii) mininum of the potential,

(iv) position of the potential minimum.

The real space pseudopotential function can be easily employed in KohnSham equation to study the surface phonons. In the local density approximation the non-diagonal elements of the dynamical matrix $D_{\alpha \beta}\left(l k, l^{\prime} k^{\prime}\right)$ can be written in terms of the response function $\mathcal{N}\left(r, r^{\prime}\right)$ as [27]:

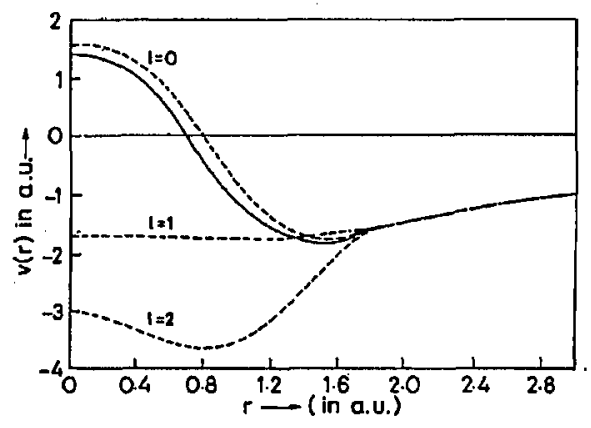

Fig. 3. Real space localised pseudopotential (solid curve) compared with the nonlocal $a b$ initio pseudopotential (dashed curve) of Ref. [2] for Al.

\section{TABLE IV}

Parameters (in a.u.) for the analytic localised pseudopotential (Eq. (4)).

\begin{tabular}{l|c|c|c|c}
\hline \hline Metals & $\alpha$ & $A$ & $B$ & $C$ \\
\hline $\mathrm{Na}$ & 0.679 & 0.838 & 2.7704 & -1.545 \\
$\mathrm{~K}$ & 0.309 & 0.678 & 0.950 & -0.423 \\
$\mathrm{Rb}$ & 0.312 & 0.922 & 0.976 & -0.515
\end{tabular}




$$
\begin{gathered}
D_{\alpha \beta}\left(l k, l^{\prime} k^{\prime}\right)=M^{-1}\left\{\iint \nabla_{\alpha} V|[r-\boldsymbol{R}(l, k)]| \mathcal{N}\left(r, r^{\prime}\right) \nabla_{\beta} V\left|\left[r-\boldsymbol{R}\left(l^{\prime}, k^{\prime}\right)\right]\right|\right. \\
\left.\quad \times \mathrm{d}^{3} r \mathrm{~d}^{3} r^{\prime}\right\}+D_{\alpha \beta}^{\text {ion }}\left(l k, l^{\prime} k^{\prime}\right) \ldots
\end{gathered}
$$

where $V(r)$ is the pseudopotential and $D_{\alpha \beta}^{\text {ion }}\left(l k, l^{\prime} k^{\prime}\right)$ represents the direct ion-ion contribution.

The phonon dispersion curves at the (100) surface of metallic sodium were calculated. The calculation is carried out for 13 layer slabs of $\mathrm{Na}$ bounded by (100) planes. The results obtained (Fig. 4) are compared with available calculation [27].

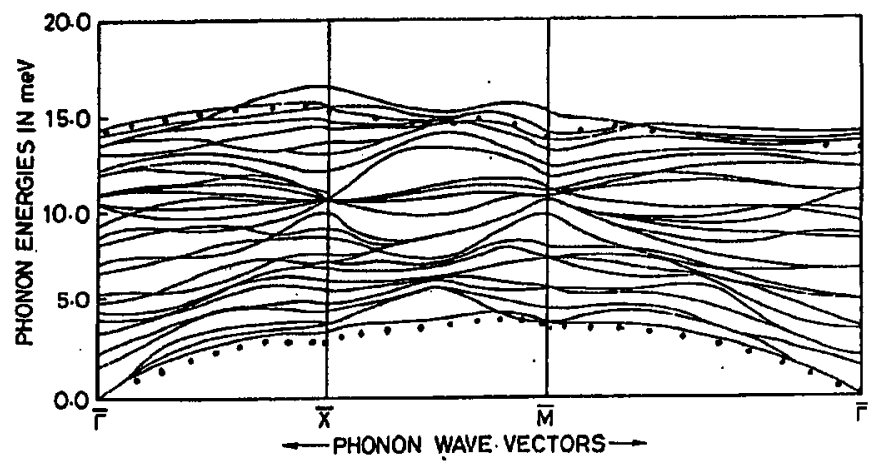

Fig. 4. Phonon dispersion curve for 100 surface of $\mathrm{Na}$ compared with some available calculated results represented by dots.

Density functional based molecular dynamics (DFMD) technique employing $a b$ initio nonlocal pseudopotentials also emerged as a powerful method to investigate structural, electronic and thermodynamic properties of inhomogeneous electron systems like molecules, clusters, liquids etc. The technique, however, requires a variety of optimization algorithms and "strategies" for orthogonalization and the calculation of nonlocal energy and forces and treatment of more than few tens of atoms turns out to be extremely expensive in terms of CPU time. There also exists an alternative simpler formulation known as orbital free method as has been used by Pearson et al. [28]. The orbital free molecular dynamics so far used local potentials only. In view of the encouraging results of the present localised scheme we believe that it might offer a reliable MD calculation. Moreover, before concluding we note that a realistic ionic pair potential derived from the localised pseudopotential as discussed earlier may be used in place of the semiempirical pair potentials [29] usually employed in Monte Carlo molecular dynamics calculations.

\section{References}

[1] V. Shah, D. Nehete, D.G. Kanhere, J. Phys., Condens. Matter 6, 10773 (1994).

[2] K. Laasonen, A. Pasquarello, R. Car, C. Lee, D. Vanderbilt, Phys. Rev. B 47, 10142 (1993). 
[3] M.M. Dacorogna, M.L. Cohen, Phys. Rev. B 34, 4996 (1986).

[4] J. Mizuki, Y. Chen, K.M. Ho, C. Stassis, Phys. Rev. B 32, 666 (1985).

[5] K.M. Ho, C.L. Fu, B.N. Harmon, Phys. Rev. B 29, 1575 (1984).

[6] M.Y. Chou, P.K. Lam, M.L. Cohen, Phys. Rev. B 28, 4179 (1983).

[7] D.R. Hamann, M. Schlüter, C. Chiang, Phys. Rev. Lett. 43, 1494 (1979); G.B. Bachlet, D.R. Hamann, M. Schlüter, Phys. Rev. B 26, 4199 (1982).

[8] D. Vanderbilt, Phys. Rev. B 41, 7892 (1990).

[9] D. Sen, Phys. Rev. B 42, 1217 (1990).

[10] M. Manninen, P. Jena, R.M. Nieminen, J.K. Lee, Phys. Rev. B 24, 7057 (1981).

[11] D. Sen, S.K. Sarkar, Phys. Rev. B 22, 1856 (1980); D. Sen, S.K. Sarkar, D. Roy, S. Sengupta, Phys. Rev. B 42, 876 (1981); Phys. Status Solidi B 115, 593 (1983);

B. Dutta, D. Sen, D. Roy, Physica B 210, 73 (1995).

[12] V.G. Vaks, A.V. Tefilov, Sov. Phys.-Solid State 19, 139 (1977); V.G. Vaks, S.P. Kravchunk, A.V. Tefilov, ibid., p. 1271.

[13] Th. Starkloff, J.D. Joannopoulos, Phys. Rev. B 16, 5212 (1977).

[14] J.P. Perdew, S.H. Vosko, J. Phys. F, Met. Phys. 6, 1421 (1976).

[15] C.M. Bertoni, O. Bisi, C. Calendra, F. Nizzoli, J. Phys. F 5, 419 (1975).

[16] V. Heine, D. Weaire, in: Solid State Physics, Eds. F. Seitz, D. Turnbull, Academic Press, New York 1970, p. 323.

[17] A. Sarkar, D. Sen, D. Roy, in: Proc. S.S.P. Symp., Varanasi, Vol. 34C, 1991, p. 93.

[18] M.L. Cohen, V. Heine, in: Solid State Physics, Eds. F. Seitz, D. Turnbull, Academic Press, New York 1970, p. 183.

[19] D. Sen, in: Proc. S.S.P. Symp., Bhopal, Vol. 31C, 1988, p. 115.

[20] W.A. Harrison, Psedopotential in the Theory of Metals, W.A. Benjamin, New York 1966, p. 136.

[21] N.W. Ashcroft, Interatomic Potential and Simulation of Lattice Defects, Plenum Press, New York 1972, p. 91.

[22] N.E. Causk, The Physics of Structurally Disodered Malter, Adam Hilger, Bristol, England 1987, p. 176.

[23] A. Sarkar, D. Sen, D. Roy, Proc. Nat. Conf. on Phonon Physics, Cochin Univ., 1993, Wiley Eastern, India, in press.

[24] K. Hoshino, H. Ugawa, M. Watabe, J. Phys. Soc. Japan 61, 2182 (1992).

[25] R. Winter, C. Pilgrim, F. Harnsel, C. Morkel, W. Glässer, J. Non-Cryst. Solids 156, 9 (1993).

[26] A. Sarkar, D. Sen, D. Roy, in: Proc. S.S.P. Symp., Jaipur, Vol. 37C, 1994, p. 95.

[27] A.G. Eguilz, A.A. Maradudin, R.F. Wallis, Phys. Rev. Lett. 60, 809 (1988).

[28] M. Pearson, E. Smargiassi, P.A. Madden, J. Phys. Condens. Matter 5, 3221 (1993).

[29] D.G. Pettifor, M.A. Ward, Solid State Commun. 49, 291 (1984).

[30] D. Sen, S.K. Sen, Phys. Status Solidi B 127, 377 (1985).

[31] W.M. Shyu, J.H. Wehling, M.R. Cordes, Phys. Rev. B 4, 1802 (1971). 